\title{
Parámetros bioquímicos enzimáticos (ALT, AST, ALP, $\gamma$-GT, LDH) en niños con leucemia linfoblástica aguda antes del tratamiento antineoplásico
}

\author{
Jeél Moya S1 , Liz Pio D²
}

\section{RESUMEN}

Objetivo: Determinar los parámetros bioquímicos enzimáticos (Transaminasa Glutámico Pirúvica (ALT), Transaminasa Glutámico Oxalacetico (AST), Fosfatasa Alcalina (ALP), Gamma Glutamiltransferasa ( $\gamma-\mathrm{GT})$, y Lactato Deshidrogenasa (LDH)) en niños con Leucemia Linfoblástica Aguda (LLA) antes del tratamiento antineoplásico.

Material y Métodos: Estudio prospectivo experimental, observacional, de corte transversal, en 30 niños con LLA entre 2 y 15 años, en diversos Centros Neoplásicos de Lima. La colecta sanguínea se realizó en tubos BD Vacutainer ${ }^{\circledR}$ de tapa color rojo, procesados en el analizador semiautomatizado BIOTEC $₫$ EMP-168, con reactivos enzimáticos de Wiener Lab Group bajo el método modificado de Szaaz y UV-Optimizado por IFCC, SSCC y SFBC. Por último, se realizó la codificación y tabulación de datos.

Resultados: El 60\% correspondió a niños y el 46,7\% corresponde a las edades entre 2 a 6 años. Los niveles séricos de AST estuvieron incrementados en el $33,3 \%$ en niños y el $50 \%$ en niñas.

Los valores séricos de ALT estuvieron incrementados en el $33,3 \%$ de niños y en el $41,7 \%$ de niñas; solamente el $25 \%$ de niñas presentaron valores incrementados de $\gamma$-GT; la ALP estuvo acrecentada en el $44,4 \%$ de niños y en el $66,7 \%$ de niñas. Por otro lado los niveles de LDH estuvieron acrecentados en el $55,6 \%$ de niños y en el $41,7 \%$ de niñas.

Conclusión: Las pruebas enzimáticas LDH, AST, ALT y ALP se encuentran aumentados en niños con LLA con respecto a los valores normales, debido al síndrome de lisis tumoral caracterizada por alteraciones electrolíticas, y como consecuencia de la destrucción masiva de células tumorales y la liberación rápida de grandes cantidades de elementos intracelulares. (Horiz Med 2015; 15(4): 52-58)

Palabras clave: ALT, AST, ALP, Y-GT, LDH, Leucemia Linfoblastica Aguda. (Fuente: DeCS BIREME).

Enzymatic biochemical parameters (ALT, AST, ALP, $\gamma$-GT, LDH) in children with acute lymphoblastic leukemia before antineoplastic treatment

\begin{abstract}
Objective: To determine the enzymatic biochemical parameters (glutamic pyruvic transaminase (ALT), glutamic oxaloacetic transaminase (AST), alkaline phosphatase (ALP), gamma glutamyltransferase ( $\gamma-G T)$, and lactate dehydrogenase (LDH)) in children with acute lymphoblastic leukemia (ALL) before cancer treatment.

Material and Methods: A prospective experimental, observational, cross-sectional study was conducted in 30 children between 2 and 15 years old, from several Neoplastic Centers in Lima. Blood collection was performed in BD red cap Vacutainer tubes, processed in the semi-automated analyzer BIOTEC $₫$ EMP-168, with Wiener Lab Group enzyme reagents under the modified method Szaaz and UV-Optimized by IFCC, SSCC and SFBC. Finally, coding and tabulation was performed. Results: $60 \%$ were boys and $46.7 \%$ are between the ages of 2-6 years. Serum levels of AST were increased by $33.3 \%$ in boys and $50 \%$ in girls. Serum ALT values were increased in $33.3 \%$ of boys and $41.7 \%$ of girls; only $25 \%$ of girls showed increased levels of $\mathrm{Y}$-GT values; ALP was increased in $44.4 \%$ of boys and $66.7 \%$ of girls. Moreover LDH levels were increased in $55.6 \%$ of boys and $41.7 \%$ of girls.

Conclusion: The enzymatic tests LDH, AST, ALT and ALP are increased in children with ALL compared to normal values due to tumor lysis syndrome characterized by electrolyte abnormalities, and as a result of the massive destruction of tumor cells and rapid release of large amounts of intracellular elements. (Horiz Med 2015; 15(4): 52-58)
\end{abstract}

Key words: ALT, AST, ALP, Y-GT, LDH, dehydrogenase, Acute Lymphatic Leukemia. (Source: MeSH NLM).

1 M.T., Dipl. en Control de Calidad en laboratorio clínico. Hospital Nacional Docente Madre Niño San Bartolomé, Lima, Perú.

2 Interna de Pregrado de Tecnología Médica en Laboratorio Clínico y Anatomía Patológica. Hospital Nacional Dos de Mayo, Lima, Perú. 


\section{INTRODUCCIÓN}

La Leucemia Linfoblástica Aguda (LLA), es la enfermedad maligna más común en niños menores de 15 años, que comprende el $85 \%$ del total de neoplasias pediátricas, con una incidencia de 34 casos por millón (1).

Esta patología es un tipo de cáncer producido por una mutación genética asociado a varios factores predisponentes, que ocasionan proliferación monoclonal anormal de células precursoras hematopoyéticas (troncales) comprometidas 0 parcialmente comprometidas de la serie linfoide y que infiltran más del $25 \%$ de la médula ósea afectando al sistema hematopoyético, primero, y conforme transcurre la historia natural de la enfermedad, invaden el torrente sanguíneo, el sistema linfático y otros órganos como la columna vertebral, testículos y el cerebro $(2,3)$.

Las características clínicas de la LLA se muestran de forma similar a los linfomas, esta puede presentarse en el momento del diagnóstico con esplenomegalia, hepatotoxicidad y linfadenopatía en aproximadamente la mitad de los pacientes. La LLA predomina ligeramente en los varones $(60 \pm 10 \%)$ en edades que oscilan entre 1 y 9 años $(1,4,5)$; considerándose este el cáncer más común, que si no se trata a tiempo tiende a agravarse rápidamente por su elevada tasa de crecimiento lítico tumoral (6).

Contrariamente, las posibilidades de curarse son satisfactorias (dos de tres lactantes) si la enfermedad se detecta a tiempo y se emplea el terapéutica adecuada dirigida hacia una lesión genética (hiperdiploidia, cromosoma Philadelphia, hipodiploidias, mutaciones de la quinasa de Janus, sobre expresión y amplificación génica, etc.) $(1,7,8)$.

Si bien, se conocen el metabolismo enzimático en la LLA, poco se sabe sobre la importancia funcional de la glicólisis, la influencia de la expresión genética en las elevadas tasas de esta ruta metabólica y sobre la expresión de los genes que codifican enzimas de la vía glicolítica en células cancerígenas (9).
Se infieren que la alta tasa de glicólisis es uno de los mecanismos más usuales para la elevada proliferación celular, por promover células grasas y limitar el consumo de oxígeno. Este proceso continuo promueve el rápido crecimiento carcinogénico (5).

Asimismo, la descontrolada expansión clonal promueve alta producción enzimática generada durante el metabolismo celular; la cuantificación de estas concentraciones son útiles para precisar ciertos diagnósticos frente a diversos fenómenos fisiopatológicos de LLA (10).

Resulta útil, entonces, las determinaciones enzimáticas de las Transaminasa Glutámico Pirúvica (TGP o ALT) y Glutámico Oxalacetica (TGO o AST); de la Fosfatasa Alcalina (ALP por sus siglas en inglés), de la Gamma Glutamil-transferasa ( $\gamma-G T)$ y Lactato Deshidrogenasa (LDH) en niños con LLA, solicitadas a nivel clínico para evaluar riesgos antes del inicio de la terapéutica, ya que las células cancerosas frecuentemente secretan enzimas que les permiten invadir tej idos circundantes, digiriendo las barreras tisulares y facilitando la migración y proliferación de las células tumorales (1). El síndrome de lisis tumoral (SLT) produce un enorme desequilibrio del medio interno al liberarse grandes cantidades de contenido intracelular al espacio intersticial e intravascular (6).

Las concentraciones bioquímicas enzimáticas son heterogéneas para estos marcadores en pacientes con LLA.

El incremento de LDH está estrechamente relacionada con lisis celular continua, así como, con proliferación anormal de clonas celulares siendo muy útil para confirmar invasión metastásica de neoplasias refractarias a tratamiento y como factor pronóstico adverso en linfomas, cáncer refractario de próstata y en LLA (11).

Además, el aumento de la tasa glicolítica está relacionada con la producción de lactato en el crecimiento proliferativo tumoral, el paso de piruvato a lactato es un punto crítico en el metabolismo del cáncer (12). 
Está demostrado que las células tumorales tienen la capacidad de un amplio metabolismo glucémico, promovido a lactato a velocidades mayores que una celular normal (13).

Además, todas las sobre expresiones génicas ocurren durante la glicólisis (11).

Esta acidosis láctica severa como complicación metabólica retarda el diagnostico de neoplasia hematológica (14). Los marcadores enzimáticos del hígado sufren alteraciones discretas en un $18 \%$ de los pacientes con LLA, evidenciados con transaminasas mayores a $100 \mathrm{U} / \mathrm{I}$, bilirrubina total mayor a 1,5 mg/dl y ALP anormal (1).

Está demostrado, que la ALT es la prueba más específica para detectar una disfunción hepática, alterada hasta en un $64 \%$ de los enfermos frecuentemente durante la fase de mantenimiento terapéutico, en la cual ocurren con frecuencia anomalías de las pruebas que miden la función hepática $(15,16)$.

Además, el tratamiento de mantenimiento de la LLA es único por su prolongada administración de dosis bajas de quimioterapia para optimizar la oportunidad de curación (17).

Por estos motivos se desarrolló la presente investigación, para determinar los parámetros bioquímicos enzimáticos (ALT, AST, ALP, y-GT, y LDH) para el manejo clínico en el cuidado de niños con LLA. Estas pruebas están incluidas, por el laboratorio clínico, en niños con LLA para la evaluación de su pronóstico, estaditaje, evolución, incremento o la asociación con lisis celular continua o con proliferación anormal de clonas celulares.

Por todo lo anteriormente mencionado el objetico de la investigación fue determinar el comportamiento de los parámetros bioquímicos enzimáticos ALT, AST, ALP, Y-GT y LDH, en niños con LLA, antes del tratamiento antineoplásico.

\section{MATERIAL Y MÉTODOS}

Tipo y diseño de investigación: Se realizó un estudio experimental, observacional, prospectivo de corte transversal, en diversos Centros Neoplásicos de Lima como unidad de análisis.

Muestra: Las muestras de suero sanguíneo la constituyeron 30 niños con diagnóstico confirmado de LLA entre 2 y 15 años de edad antes del tratamiento con quimioterapia, lo cual representó entre el 25 a 30\% del total de pacientes en esta patología. Se obtuvieron gracias al apoyo del personal médico de los departamentos de patología de diferentes Centro Neoplásicos de Lima.

Técnica de recolección de datos y procesamiento de la muestra: Se solicitó autorización a través del consentimiento informado a cada paciente y/ o encargado que fue parte del estudio.

Se coordinó con los médicos tratantes la colecta de muestra sanguínea de los niños con LLA, en tubos BD Vacutainer ${ }^{\circledR}$ (Franklin Lakes, New Jersey) de $3 \mathrm{ml}$ de tapa color rojo con activador de coagulo, procesados como señala la guía CLSI H03-A6 (18).

Se realizó el análisis serológico en el analizador bioquímico semiautomatizado BIOTEC $\circledast$ EMP-168 (EMP Medical Headquarter, Shenzhen) a cada una de las muestras obtenidas. Por último, se realizó la codificación y tabulación de datos.

Los reactivos enzimáticos fueron de Wiener Lab Group. (Rosario, Argentina) para ALT y AST bajo el método UV-Optimizado por la IFCC -Federación Internacional de Química Clínica y Medicina de Laboratorio- $(19,20,21)$.

La $\mathrm{Y}$-GT por el método de modificado de Szaaz $(22,23)$, ALP bajo el método cinético optimizado por DGKC y SSCC $(24,25,26)$ y el método UV-Optimizado por SFBC (Sociedad Francesa de Biología Clínica) para LDH (27).

Técnica de Análisis de Datos: El análisis se realizó mediante la estadística descriptiva como la distribución de frecuencias. Dichos resultados se registraron en un instrumento de recolección 
de datos, posteriormente se tabuló en una base de datos para analizarse con el programa informático Statistical Package for Social Sciences (SPSS) versión 20,0 para Windows.

Ética: Los aspectos éticos en la investigación están garantizados por la confiabilidad y el resguardo que tendrá el investigador sobre la información obtenida, la cual se mantendrá en anonimato y solamente se utilizará para esta investigación.

Se informo a los participantes y/o representantes sobre los beneficios y posibles perjuicios que la extracción sanguínea puede tener sobre el bienestar y su salud, para cubrir los objetivos especificados en el proyecto, mediante consentimiento informado.

Limitaciones: Primero, no realizamos el control enzimático durante el tratamiento con quimioterápicos para evaluar su comportamiento. Segundo, no se correlacionaron las concentraciones bioquímicas enzimáticas con los subtipos de LLA. Tercero, sólo se evaluaron los parámetros bioquímicos enzimáticos señalados. Por último, el tratamiento farmacológico no estuvo asociado a las variantes genéticas diagnosticadas de pacientes con LLA.

\section{RESULTADOS}

Tabla 1. Pacientes con LLA discriminados por sexo

\begin{tabular}{lcc} 
Sexo de los pacientes & Cantidad & $\%$ \\
\hline Masculino & 18 & $60.0 \%$ \\
\hline Femenino & 12 & $40.0 \%$ \\
\hline Total & 30 & $100.0 \%$ \\
\hline
\end{tabular}

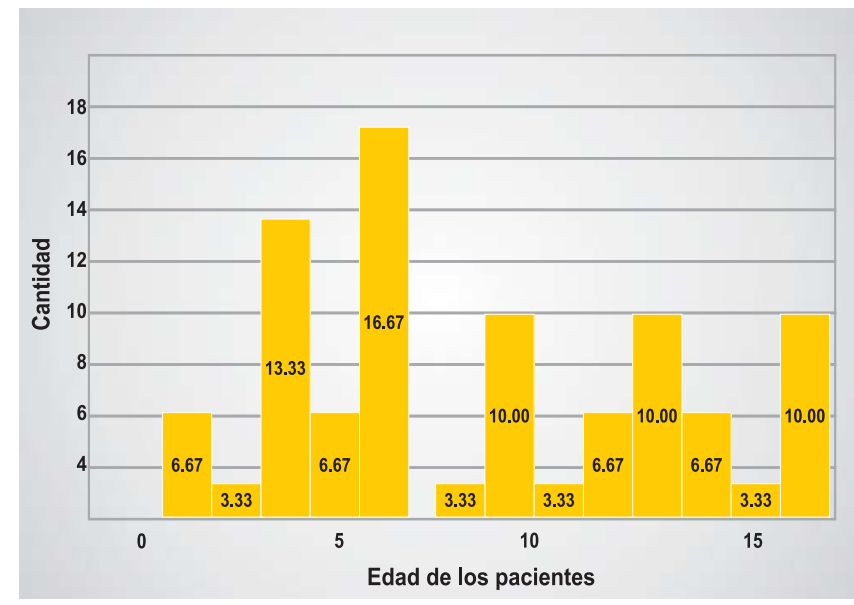

Figura 1. Edades de los pacientes con LLA

Tabla 2. Resultados de los parámetros bioquímicos enzimáticos

\begin{tabular}{|c|c|c|c|c|c|}
\hline \multirow{3}{*}{\multicolumn{2}{|c|}{$\begin{array}{c}\text { Valores de los parámetros } \\
\text { bioquímicos }\end{array}$}} & \multicolumn{4}{|c|}{ Sexo de lo pacientes } \\
\hline & & \multicolumn{2}{|c|}{ Masculino } & \multicolumn{2}{|c|}{ Femenino } \\
\hline & & Cantidad & $\%$ & Cantidad & $\%$ \\
\hline \multirow{3}{*}{$\begin{array}{l}\text { Valores de ALT } \\
\text { (TGP) }\end{array}$} & Bajo & 2 & $11.1 \%$ & 0 & $0.0 \%$ \\
\hline & Normal & 10 & $55.6 \%$ & 7 & $58.3 \%$ \\
\hline & Alto & 6 & $33.3 \%$ & 5 & $41.7 \%$ \\
\hline \multirow{3}{*}{$\begin{array}{l}\text { Valores de AST } \\
\text { (TGO) }\end{array}$} & Bajo & 1 & $5.6 \%$ & 0 & $0.0 \%$ \\
\hline & Normal & 11 & $61.1 \%$ & 6 & $50.0 \%$ \\
\hline & Alto & 6 & $33.3 \%$ & 6 & $50.0 \%$ \\
\hline \multirow{3}{*}{ Valores de ALP } & Bajo & 0 & $0.0 \%$ & 0 & $0.0 \%$ \\
\hline & Normal & 10 & $55.6 \%$ & 4 & $33.3 \%$ \\
\hline & Alto & 8 & $44.4 \%$ & 8 & $66.7 \%$ \\
\hline \multirow{3}{*}{ Valores de $\gamma-G T$} & Bajo & 0 & $0.0 \%$ & 1 & $8.3 \%$ \\
\hline & Normal & 181 & $100.0 \%$ & 8 & $66.7 \%$ \\
\hline & Alto & 0 & $0.0 \%$ & 3 & $25.0 \%$ \\
\hline \multirow{3}{*}{ Valores de LDH } & Bajo & 1 & $5.6 \%$ & 0 & $0.0 \%$ \\
\hline & Normal & 7 & $38.9 \%$ & 7 & $58.3 \%$ \\
\hline & Alto & 10 & $55.6 \%$ & 5 & $41.7 \%$ \\
\hline
\end{tabular}




\section{DISCUSIÓN}

El fundamento racional de la medición de actividades enzimáticas en plasma o suero humano se basa en la premisa que han tenido lugar cambios en un tejido u órgano específico (28).

Ya que, los sistemas enzimáticos están alterados frecuentemente en la mayoría de neoplasias hematológicas (29).

La determinación de estos catalizadores proteicos en muestras clínicas de pacientes con LLA sirve para conocer el órgano específico en un proceso patológico (15).

Así, en esta investigación, la ALT estuvo elevada circunspectamente en unos 33,3\% para el género masculino y $50 \%$ para el femenino respecto al total de pacientes estudiados en comparación con Gonzales A, et al., donde la ALT fue la prueba más específica para detectar una disfunción hepática y estuvo elevada en el 63,7\% de 124 pacientes (15).

El $33,3 \%$ de varones y el $45,8 \%$ de mujeres presentaron hipertransaminasemia, con coeficiente anormal de AST/ALT en promedio mayor a 1,3, que refleja hepatopatía por neoplasia primaria, coincidentes con los resultados de Campbell BM, et al. $(1,6)$.

Así también, la enzima que se elevó con mayor frecuencia fue la LDH; sin embargo, no podemos considerar el aumento de esta última como un indicador de enfermedad hepática, ya que no determino el tipo de isoenzimas.

La elevación de esta enzima no se correlaciona con la extensión de la necrosis hepatocelular y no tiene valor pronóstico, pero puede ser un indicador precoz de disfunción del órgano (30). Además, la elevación de LDH está relacionada con síndromes linfoproliferativos, donde se producen ascensos moderados hasta en un $60 \%$ de los casos, en esta investigación $55,6 \%$ de mujeres y $41,7 \%$ de varones con LLA tuvieron valores incrementados (>230 U/l).

El incremento de LDH está más asociado con lisis celular. El SLT es un conjunto de complicaciones metabólicas asociado frecuentemente a neoplasias malignas que tienen una alta fracción de crecimiento y por consiguiente una gran susceptibilidad a los medicamentos citotóxicos.

Además, los neoplasias malignas tienen la capacidad de metabolizar la glucosa a lactato en velocidades mucho mayores que las células normales, como consecuencia dejan de ser la principal fuente de carbonos para la respiración aeróbica $(12,13,14)$.

Existe una correlación entre ALP y $\mathrm{Y}$-GT para confirmar hepatopatías, cuando hay incrementos de ALP sobre los $170 \mathrm{U} / \mathrm{l}$. Los incrementos de ALP en la infancia y adolescencia son ocasionados por aumento de la actividad osteoblástica por crecimiento de la fracción ósea; las elevaciones patológicas se deben ocasionalmente a neoplasias con origen óseo, medula ósea (6).

El $66,7 \%$ de mujeres y el $44 \%$ de varones presentaron incrementos de ALP sobre los 177 U/l según SSCC, coincidentes con los últimos estudios sobre LLA (24, 25,30 ).

Continuamente, los valores séricos normales de Y-GT son más elevados en los hombres (hasta 40 $\mathrm{U} / \mathrm{dl}$ ) en que en las mujeres (hasta $28 \mathrm{U} / \mathrm{dl}$ ), esto coincide con nuestros resultados mostrados en la Tabla 3.

Solo el $25 \%$ de mujeres mostraron valores sobre los $32 \mathrm{U} / \mathrm{dl}$, que señala hepatopatía asociado a infancia o por osteopatía neoplásica, siendo este marcador, muy sensible, pero poco específico (6).

Campbell BM, et al. señaló que la distribución de LLA por sexo muestra una proporción de varones mayor que la descrita habitualmente $(62 \%$ contra $55 \%$ ), esto puede estar afectado por la proporción de pacientes incluidos en la investigación, por lo que resulta indeterminado porcentualizar la prevalencia de niños con LLA en esta investigación (1).

La LLA es un enfermedad maligna de los tejidos formadores de sangre, caracterizada por una expansión clonal, rápida y descontrolada de linfoblastos, que requiere mayores estudios en química hemática, instrumento de utilidad clínica, para establecer alcances, limitaciones y valores 
clínicos en todas las etapas del diagnóstico, tratamiento y control de pacientes con LLA.

En conclusión, esta investigación evaluó el comportamiento de los parámetros bioquímicos enzimáticos ALT, AST, ALP, Y-GT y LDH en niños con LLA antes del tratamiento antineoplásico.

Del total de pacientes estudiados el $60 \%$ correspondió al género masculino y el $46,7 \%$ de dichos pacientes corresponde a las edades que oscilan entre 2 a 6 años.

De los pacientes estudiados con LLA antes del tratamiento antineoplásico el $33,3 \%$ en niños y el $41,7 \%$ en niñas, presentaron concentraciones séricas de ALT incrementadas, mientras que el $33,3 \%$ en niños y el $50 \%$ en niñas tuvieron concentraciones séricas incrementadas para AST En ambos se presento hipertransaminasemia, con coeficiente anormal de AST/ALT.

Solamente el $25 \%$ de niñas con LLA antes del tratamiento antineoplásico mostraron valores séricos de $\gamma$-GT sobre los $32 \mathrm{U} / \mathrm{dl}$. El 44,4\% en niños y el $66,7 \%$ en niñas, tuvieron niveles acrecentados de ALP, en promedio mayores a $177 \mathrm{U} / \mathrm{l}$.

Tanto ALP como $\gamma$-GT son dictámenes que deben someterse a consideración clínica para una lectura e interpretación de resultados adecuada.

Finalmente, los niveles séricos de LDH estuvieron incrementados en $55,6 \%$ de niños y los $41,7 \%$ de niñas con LLA antes del tratamiento antineoplásico (en promedio $>230 \mathrm{U} / \mathrm{l}$ ), relacionados principalmente con el síndrome linfoproliferativo.

\section{Agradecimiento}

Expresamos nuestro agradecimiento al Lic. Ronald Torres Martínez y Lic. TM. Víctor Rojas Zumaran por su asesoría metodológica y estadística.

\section{Fuentes de financiamiento}

Este trabajo fue financiado por los autores.

\section{Conflictos de interés}

Los autores declaran no tener ningún conflicto de interés

\section{REFERENCIAS BIBLIOGRÁFICAS}

1. Campbell BM, Ferreiro CM, Tordecilla CJ, et al. Leucemia linfoblástica aguda. Características al diagnóstico en 100 niños. Rev chil pediatr 1999; 70(4): 288-293

2. Msefer AF. Diagnóstico precoz del cáncer en el niño. Manual de formación para los médicos del primer nivel de atención. Asociación “L’Avenir” Rabat-Marruecos.1ª edición. Perú: Reliza S.R.L.; 2010.

3. Beutler E, Lichtman M, Coller B. Williams Hematología. 1a edición. España: Marbán Libros, S. L.; 2005.

4. Anzola DC, Suarez A, Feliciano J. Estado nutricional en niños con leucemia linfoblástica aguda en el Instituto Nacional de Cancerología de Bogotá, Colombia 2007-2011. [Tesis]. Bogotá: Universidad de la Sabana. Facultad de Medicina; 2011.

5. Eigenbrodt, E., Fister, P., Reinacher, M. New perspectives in carbohydrate metabolism in tumor cells. In: Reitner R. Regulation of carbohydrate metabolism. Boca Raton: CRC Press; 1985: 141-179.

6. Balcells A, Prieto J, Yuste J. La clínica y el laboratorio: interpretación de análisis y pruebas funcionales. Exploración de los síndromes. Cuadro biológico de las enfermedades. 20ava edición. España: Masson; 2006.

7. Fallabela FF, Cuéllar AF. Hematología. Fundamentos de medicina. 6a edición. Madrid: Cooperación para Investigaciones Biológicas; 2005; 118-24.

8. Ching-Hon P. Acute lymphoblastic leukemia. In: Lichtman MA, Beutler E, Kaushansky K, et al. eds. Acute lymphoblastic leukemia. Williams Hematology. 8va ed. New York, NY: McGraw Hill Professional; 2010: 93.

9. Lassaletta AA. Leucemia: Leucemia Linfoblastica Aguda. Servicio de Hemato-Oncología Pediátrica. Hospital Universitario Niño Jesús, Madrid. Pediatr Integral 2008;12(6):551-558. 
10. Abbas A, Kumar V, Fausto N. Robbins. Patología humana. 8a edición. España: Elsevier; 2008.

11. Aranda TE. Interpretation of lactate dehydrogenase. Rev Bol Ped 2010;49(2): 132-134.

12. Gonzales CC, Gonzales RG, Guerinoni ED, Tubeh CR. Sobreexpresión de genes de las enzimas de la vía glicolítica en células cancerígenas. Acta Med Per 2007; 24(3): 187-96.

13. Warburg 0 . On respiratory impairment in cancer cells. Science. 1956; 124(3215): 269-270.

14. Loja D, Vilca M, Postigo R, Torres A, Alvizurri J. Acidosis láctica severa y leucemia aguda. An Fac Med UNMSM. 2004; 65 (1):49-55.

15. González A, Machín S, Castañeda C, Svarch E, Roque MC. Estudio de las alteraciones hepáticas en un grupo de pacientes con leucemia aguda linfoblástica. Rev Cubana Hematol Inmunol Hemoter 1997; $12(1)$.

16. Suárez LB, Carnot JU, Arenas de Castro R, Muñío JP, Martínez MC, et al. Leucemia Aguda en pacientes de 60 años. Hospital clínico quirúrgico "Hermanos Almejeiras". Servicio de Hematología. Rev Cubana Med 2003; 42(1):18-26.

17. Ortiz CA, Cortez CE, Nochez TM. Toxicidad de la quimioterapia en pacientes con diagnóstico de Leucemia Linfocítica Aguda, Leucemia Mielocítica Aguda y Linfomas. [Tesis]. Guatemala: Universidad de San Carlos de Guatemala. Facultad de Ciencias Médicas; 2011.

18. Clinical and Laboratory Standard Institute. H03-A6. Procedures for the collection of diagnosis blood Specimens by Venipunture. 2007; 27(26).

19. Bergmeyer HU, Bowers GN Jr, Horder M, Moss DW. Provisional recommendations on IFCC methods for the measurement of catalytic concentrations of enzymes. Part 2. IFCC method for aspartate aminotransferase. Clin Chim Acta. 1976; 70(2):F19-29.

20. Bergmeyer HU. IFCC methods for the measurement of catalytic concentrations of enzymes Part 3. IFCC method for alanine aminotransferase (1-alanine:2oxoglutarate aminotransferase) Clin Chem Acta 1980;105(1):147.

21. Burtis R, Ashwood E. Tietz textbook of Clinical Chemistry. 3rd Edition. Massachusetts: Saunders Co., 1999.

22. Szasz G. A kinetic photometric method for serum gamma-glutamyl transpeptidase. Clin Chem. 1969;15(2):124-36.

23. The Commitee on Enzymes of the Scandinavian Society for Clinical Chemistry and Clinical Physiology. Recommended method for the determination of g-glutamyltransferase in blood. Scand J Clin Lab Invest 1976;36:119-125.

24. Scandinavian Society for Clinical Chemistry and Clinical Physiology. Recommended methods for the determination of four enzymes in blood. Scand J Clin Lab Invest 1974;33(4):291-306.

25. Anon I. Optimized standard method for quantitative determination of alkaline phosphatase. Z. Klin. Chem. Klin. Biochem. 1970;8: 658.

26. Anon I. Optimized standard method for quantitative determination of alkaline phosphatase. Z. Klin. Chem. Klin. Biochem. 1972; 10: 182.

27. Enzymology Commission: Société Française de Biologie Clinique. Recommendations for determining the catalytic concentration of lactate dehydrogenase in human serum at $30^{\circ} \mathrm{C}$ (document F). Ann Biol Clin 1982;40:160-4.

28. Bernard JH, et al. El laboratorio en el Diagnostico Clínico. 20a edición. España: Marban Libros S.A.; 2005.

29. Pui CH. Acute lymphoblastic leukemia. In: Beutler E, Lichtman MA, Coller BS, Kipps TJ, Seligsohn U, editors. Williams Hematology, 6ta Ed. New York: McGrawHill; 2001: 1141-61.

30. Stefan CD, Galindo RC. Pediatric Hematology-Oncology in Countries with Limited Resources. New York, NY: Springer; 2014.

\section{Correspondencia:}

Moya Salazar, Jeel J.

Dirección: Jr. Pacifico 957. Lima 07. Lima, Perú.

Teléfono: +051 19860149854 .

Correo electrónico: jeelms@outlook.com 